\title{
Laboratory simulation of LNAPL spills and remediation in unsaturated porous media using the image analysis technique: a review
}

\begin{abstract}
Leaking from underground storage and surface spills of hydrocarbon sources can cause serious nonaqueous phase liquid (NAPL) contamination in subsurface environments. The toxic compounds of chemicals have made field study impracticable and it has been replaced by laboratory and numerical simulations. This paper introduces the methodology for twodimensional light nonaqueous phase liquid (LNAPL) flow behavior and remediation experiments using the image analysis technique (IAT). The LNAPL flow behavior experiments are divided into qualitative and quantitative infiltration and redistribution experiments, with and without numerical modeling. The laboratory setup for the quantitative experiments emphasizes the sand tank fabrication, sand packing techniques, typical porous media properties, and the selection of the LNAPL source. In this paper, several methods of enhanced remediation experiments are discussed to explain how LNAPL extraction was carried out for remediation. The requirements and image processing in the IAT are also highlighted from the existing researches. From the discussion, this nondestructive and nonintrusive technique can provide safer and larger coverage of regions for saturation imaging of LNAPL distribution in porous media compared to other techniques. Overall, this paper discusses the laboratory works to produce a highly reliable saturation imaging and current visualization technique for characterizing and analyzing NAPL migration in 2-D aquifer models.
\end{abstract}

Keyword: Nonaqueous phase liquid; Multiphase flow; Remediation; Image analysis technique; 2-D laboratory model 